\title{
DESIGN OF GARMENTS USING ADAPTABLE DIGITAL BODY MODELS
}

\author{
DOI: $10.35530 /$ TT.2021.09
}

\author{
A. Rudolf ${ }^{1 *}$, Z. Stjepanovićn ${ }^{1}$ A. Cupar ${ }^{2}$ \\ ${ }^{1}$ Institute of Engineering Materials and Design, Faculty of Mechanical Engineering, University of \\ Maribor, Slovenia \\ (E-mail: andreja.rudolf@um.si, zoran.stjepanovic@um.si) \\ ${ }^{2}$ Mechanical Engineering Research Institute, Faculty of Mechanical Engineering, University of \\ Maribor, Slovenia \\ (E-mail: andrej.cupar@um.si)
}

\begin{abstract}
In recent years, the $3 D$ design software has been mostly used to improve the garment design process by generating virtual 3D garment prototypes. Many researchers have been working on the development of $3 D$ virtual garment prototypes using $3 D$ body models and involving the $3 D$ human body scanning in different postures. The focus of research in this field today relies on generating a kinematic $3 D$ body model for the purposes of developing the individualized garments, the exploration of which is presented in this paper. The discussed area is also implemented in the Erasmus+ project OptimTex - Software tools for textile creatives, which is fully aligned with the new trends propelled by the digitization of the whole textile sector. The Slovenian module focuses on presenting the needs of digitization for the development of individualized garments by using different software tools: 3D Sense, PotPlayer, Meshroom, MeshLab, Blender and OptiTex. The module provides four examples: 3D human body scanning using 3D photogrammetry, 3D human body modelling and reconstruction, construction of a kinematic $3 D$ body model and $3 D$ virtual prototyping of individualized smart garments, and thus displays the entire process for the needs of $3 D$ virtual prototyping of individualized garments. In the OptimTex project, the 3D software Blender was used to demonstrate and teach students how to construct the "armature" of the human body as an object for rigging or the virtual skeleton for a $3 D$ kinematic body model, using the knee as an example.
\end{abstract}

Keywords: 3D body model, 3D scanning, kinematic 3D body model, virtual prototyping of garments

\section{INTRODUCTION}

Digital transformation can be stated as the ultimate challenge for the fashion industry. In response to the pressure for growth and cost efficiency, many brands have started a series of initiatives to improve their speed to market and to implement sustainable innovation in their core product design, manufacturing, and supply chain processes. The role of the consumer has shifted from one of passive observance to enabled dominance. The vast majority of consumers use digital channels before, during or after making their purchases [1]. The fourth industrial revolution brings a lot of potentials, but also challenges. So, companies in the apparel industry are already facing a major transformation, greatly aided by effective digitalization. Virtual prototyping of garments can be termed as one of the most important parts of digitalization of the entire process. The purpose of 3D virtual prototyping is to create a virtual garment model instead of developing a real clothing product. For this, we have to 
develop and employ digital body models. These topics are being investigated by some universities and research institutions that are colaborating in different research programmes, one of these being the EC Erasmus+ programme.

Goals and objectives of the new Erasmus+ project OptimTex - Software tools for textile creatives are fully aligned with the new trends propelled by the digitalisation of the whole textiles sector. OptimTex is already the fourth in the row of the successful projects devoted to e-learning in the wider scope of textiles. The projects are carried out by the consortium, consisting of the Universities and Institutes from different European countries [2].

OptimTex is a higher education project with underlined multidisciplinary concept. Connections between modern software applications and textiles are being investigated and explored. The main outputs of the project are: (1) Course of software applications for textiles, (2): Instruments for applying software solutions within textile enterprises, (3) E-learning tool and courses organization. The Slovenian Team has developed its module on Design and modelling of garments by 3D scanning software and CAD/PDS software. The contribution is focused on design of garments using adaptable digital body models, a contemporary topic, which is fully described in the module.

\section{DIGITAL BODY MODELS}

In today's digital age, the development of garments is increasingly taking place with the help of advanced technologies for 3D scanning of the human body and 3D CAD systems for the design, simulation, and visualization of clothing on virtual 3D body models. Their breakthrough is geared towards supporting the development of new advanced personalized products, such as garments for physically impaired persons, persons with physical deformities, the elderly or, for example, athletes, workers etc. who in carrying out various activities occupy specific postures, as well as for every individual who wants custom made garments. 3D body models provided by commercial 3D CAD systems for simulating and visualizing garments on virtual 3D body models are still a problem in this area. By using them we can adjust the body dimensions, muscles and postures to a certain extent. The studies showed that the use of advanced technologies is of paramount importance for the development of garments in terms of ergonomic and functional needs of the individual in order the garment properly fits the body [3-11].

3D body models of individuals can be obtained by 3D scanning of persons in the standard posture or in many other postures, which can be imported into 3D CAD systems for the development of personalized garments. Alternatively, the scanned digital record of the individual can be upgraded to a kinematic 3D body model, of which the body postures can be adjusted according to the needs during development and prototyping of garments for individuals.

The beginnings of the development of a kinematic 3D body model for virtual clothing prototyping go back years and coincide with the development of computer graphics in the field of human body kinematics and the animation of virtual garments, where from the very beginning rigged human body models also emerged [12,13] to improve deformable virtual humans [14].

A kinematic human body model intended for 3D garments development was presented by Leipner A. and Krzywinski S. [15], which consists of the anatomical structure of the human body or skeleton, muscles and skin, Figure 1. Modeling and animation of 3D body model was carried out using the Autodesk Maya 2012 software and Maya plug-in MuscleCreator. An inverse kinematics animation was created to study the proper deformation of the leg surface during the movement of the leg (figure 1). In the study by Kozar et al [16], 
Blender software was used to design an adaptable 3D body model intended for the development of clothing for people with limited physical abilities (figure 2). This study shows the importance of using different tools to achieve smooth 3D mesh deformation and the influence they have on the body perimeters.
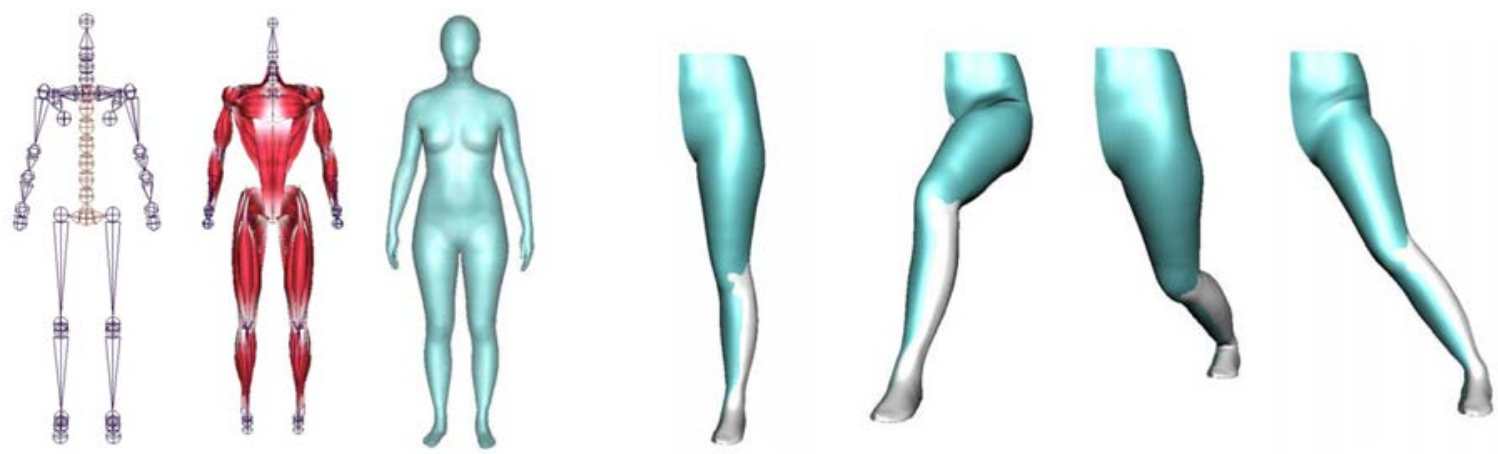

Figure 1. Kinematic 3D human body model construction: skeleton, muscles, skin (left) and animation of leg movement (right) [15].
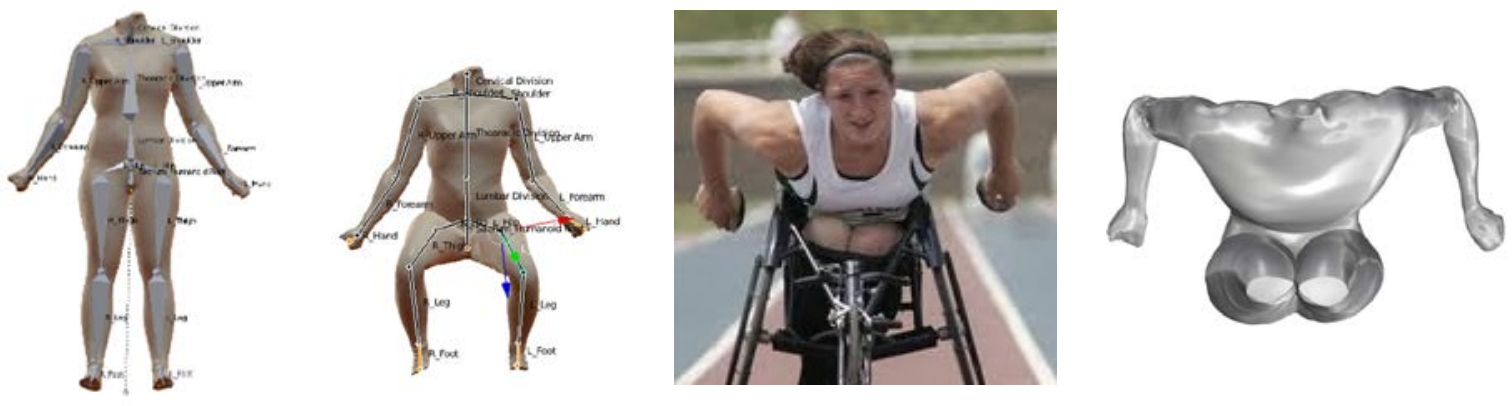

Figure 2. Designing a 3D kinematic body model and fitting it to a wheelchair racing athlete [16].

Other research has also shown that there is currently no 3D kinematic human body model that represents realistic body deformations during movement, particularly in the elbow, knee and hip joints $[17,18]$. Examined were four methods, linear blend skinning in the simulation software Clo3d, auto-rigging of 3D scans on the online service Maximo, skinned multi-person linear human model and anatomical simulation using the plugin Ziva Dynamics, which also indicated improper deformation of the body mesh during bending in the elbow, knee, and hip joint regions. As an example of working with Clo3d a 3D human body scan conversion into an avatar adapted the body shape is shown on figure 3.

A new process for construction of a kinematic 3D body model using Blender 3D software can be found in the research by Klepser A. and Pirch K. [19]. There, a comparison between the three-dimensionally software-provided avatars and 4D scans of real test persons was carried out. Figure 4 shows that despite carefully performed 3D human body scanning of the tested person and mesh processing during kinematic 3D body model generation, difficulties remained due to incorrect mesh deformations during posture adjustments. In addition, 4D scans of the tested person's movements were taken as a starting point for the creation of a new kinematic 3D body model, the accuracy of which was improved. 


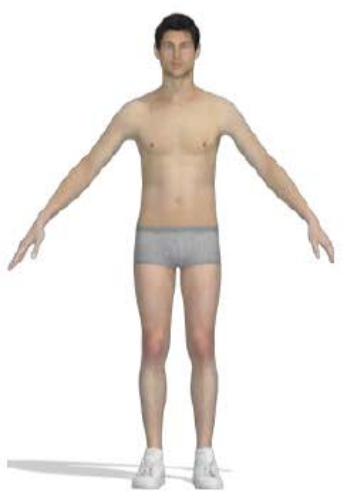

a
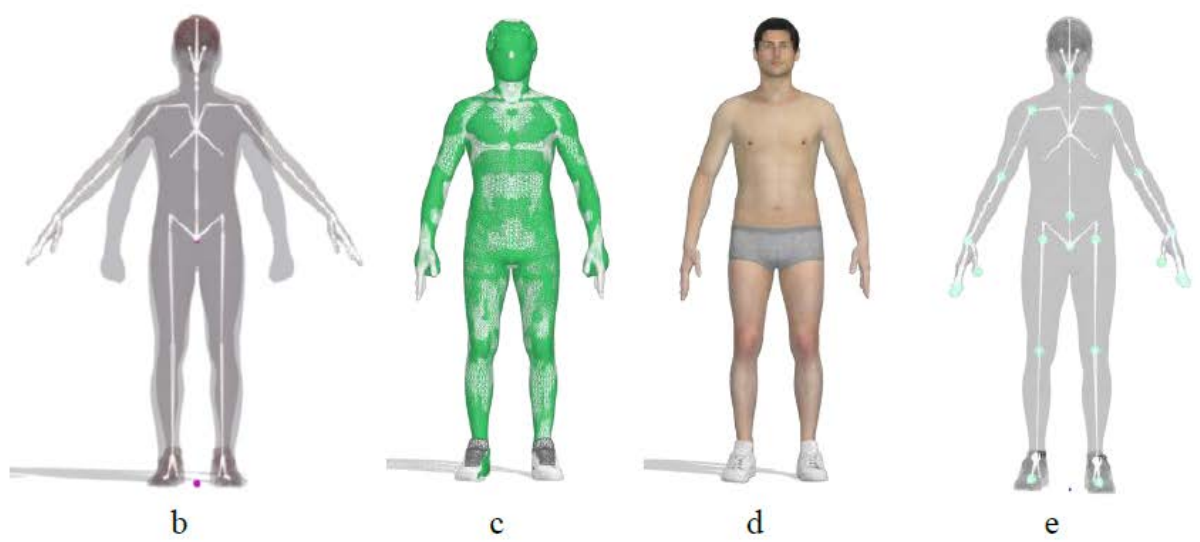

Figure 3. Converting a 3D body scan to an avatar: a - Clo avatar; b - aligned 3D body scan and Clo avatar; c - aligned avatar joints; $\mathrm{d}$ - converted avatar; e - 3D body scan with joints $[17,18]$.
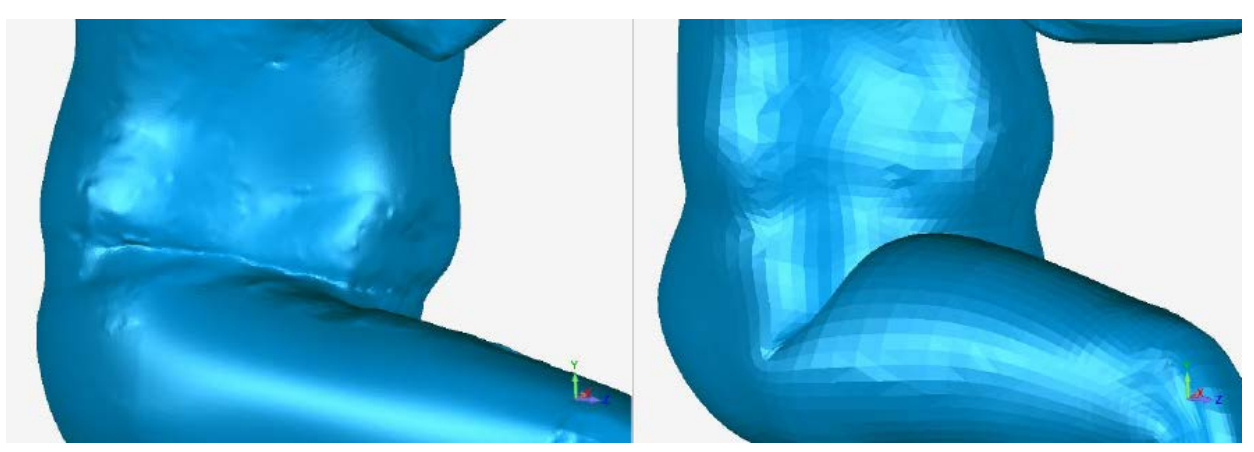

Figure 4. Leg's flexsion pose of a scanned 3D body model (left) and generated kinematic 3D body model (right) [19].

Research trends in the development of a kinematic 3D body model for the purpose of virtual prototyping of individualized garments have been transferred to the OptimTex Erasmus+ project with the aim of providing students with the full range of new digital skills and knowledge for virtual development of individualized garments.

\subsection{OptimTex Module 3 - Design and modelling of garments by 3D scanning software and CAD/PDS software}

The module focuses on presenting the needs of digitization for the development of personalized clothing by using different software tools: 3D Sense, PotPlayer, Meshroom, MeshLab, Blender and OptiTex.

The module provides four examples:

1. 3D human body scanning using the 3D photogrammetry,

2. 3D human body modelling and reconstruction,

3. Construction of a kinematic 3D body model and

4. 3D virtual prototyping of personalized smart garments 
and thus displays the entire process for the needs of 3D virtual prototyping of individualized garments.

Each example consists of the related theory, the application of software tools and some multiple-choice questions for self-assessment of the acquired knowledge.

The example of constructing a 3D kinematic body model from a 3D body model obtained by scanning and reconstructing a digitized body shows its creation through the acquisition of basic knowledge about:

1. the human body joint types divided according to their mechanics: pivot, hinge, ball-and-socket, condyloid, saddle and plane joints, which are important for skeleton generation and joints movements,

2. Blender 3D software which was used to demonstrate and teach students how to design the human body skeleton as an object for rigging or as a virtual skeleton for a kinematic body model, using the leg and flexion/positioning of the knee as examples.

The students will learn how to design a kinematic 3D model of leg's lower part and its skelton as two bones, positioned within a 3D mesh of the leg as a junction in the knee joint (hinge joint; move in the Y axis), which enables manipulation and deformation of a mesh by moving bones in the knee joint, Figure 5 .

The prepared course contains study material richly supported by video content that helps students in their creative work with software.

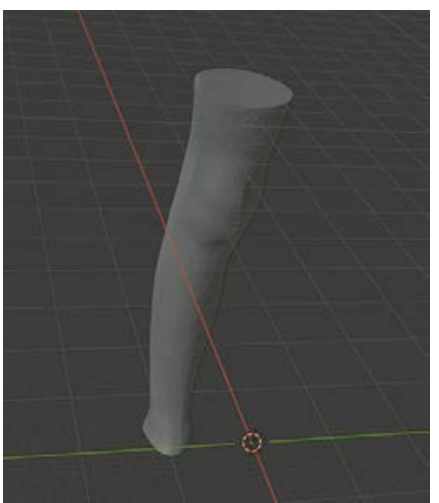

$\boldsymbol{a}$

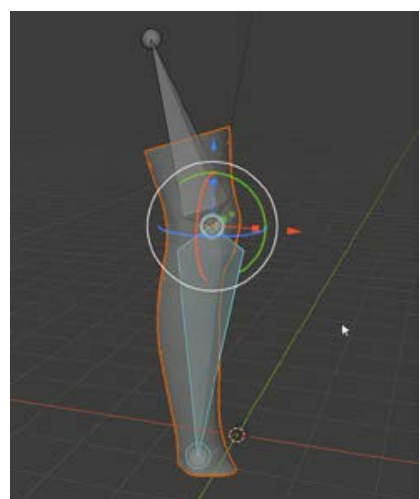

b

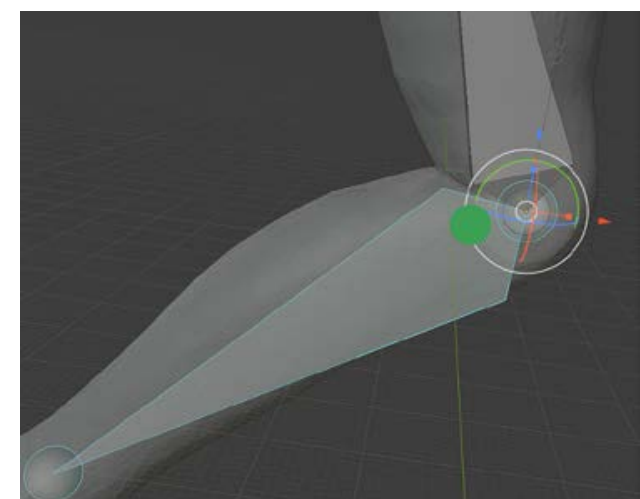

C

Figure 5. Designing of a kinematic 3D leg model: a -scanned 3D leg model; b - skeleton; $\mathrm{c}$ - kinematics of the rigged 3D model

\section{DESIGN OF GARMENTS USING KINEMATIC 3D BODY MODELS}

Recognitions that scanned 3D human body models in standard scan posture are not always suitable for the development of individualized garments, such as sports, medical and protective clothing, indicate the needs to use kinematic 3D body models. Indeed, garments that fit a standard scan posture can be very uncomfortable when performing daily tasks including walking, sitting, or reaching, and even more when performing sports or work activities. A garment design in a standard scanning posture is shown in figure 6 , a, while the developed pattern designs for speed-skating trousers and rowing dress using a kinematic 3D body model is shown in figure $6, \mathrm{~b}$ and c [15].

In the OptimTex Erasmus+ project the last example of the module - Design and modelling of garments by $3 D$ scanning software and CAD/PDS software - the development of individualized smart garment for a sitting posture of people with immobile lower extremities was presented, figure 7. Using the OptiTex PDS 3D software, the development of the pants' 
pattern design on the kinematic 3D body model is shown from the point of view of correct positioning of heating elements, temperature sensors and conductive paths for acquisition and regulation of data when measuring pants' microclimate temperature and regulating the microclimate to the desired temperature.

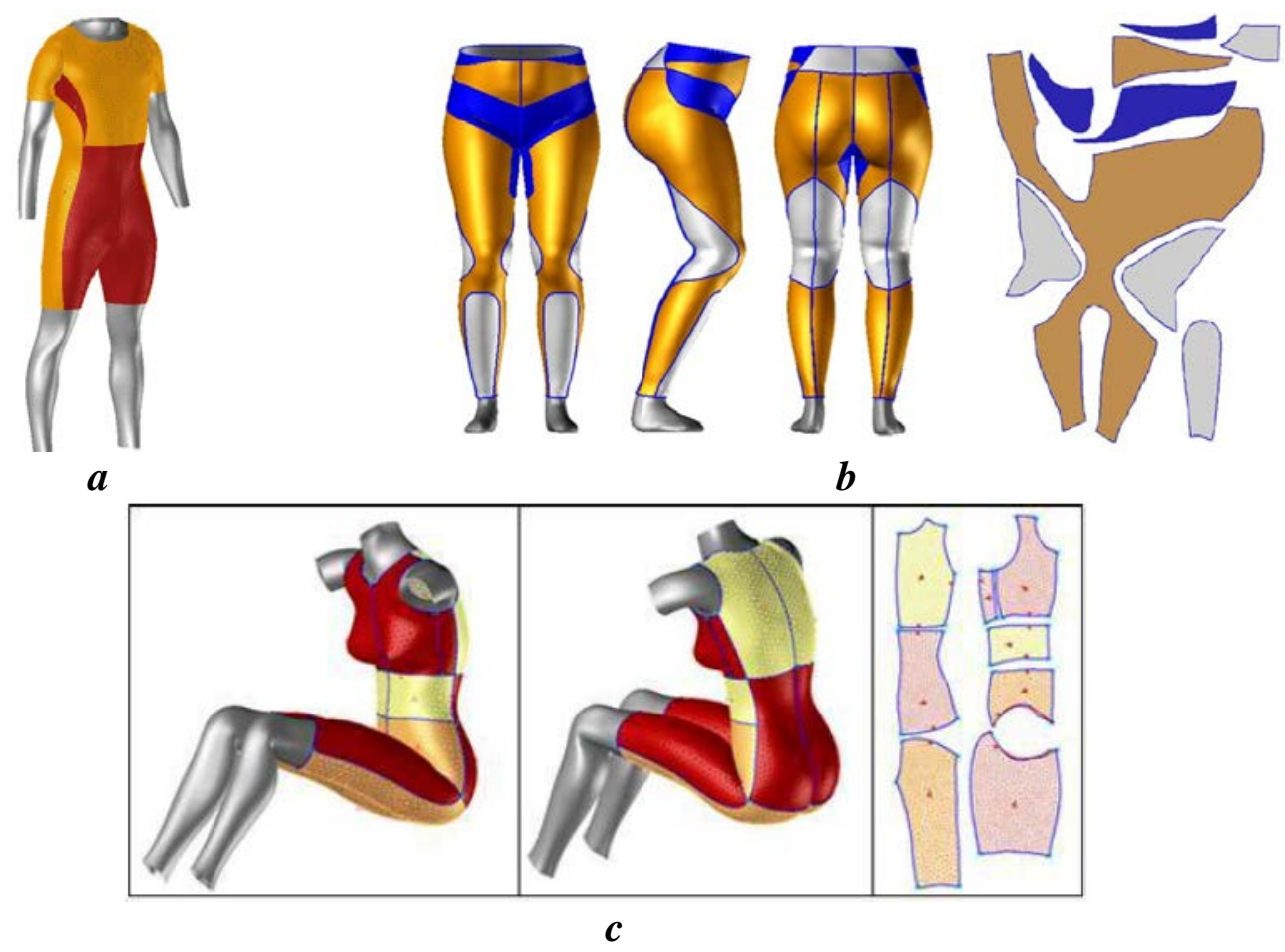

Figure 6. Garment pattern design in standard scanning posture: a - development of pattern pieces for speed-skating trousers; b - rowing dress; c - on a kinematic 3D body model [15]
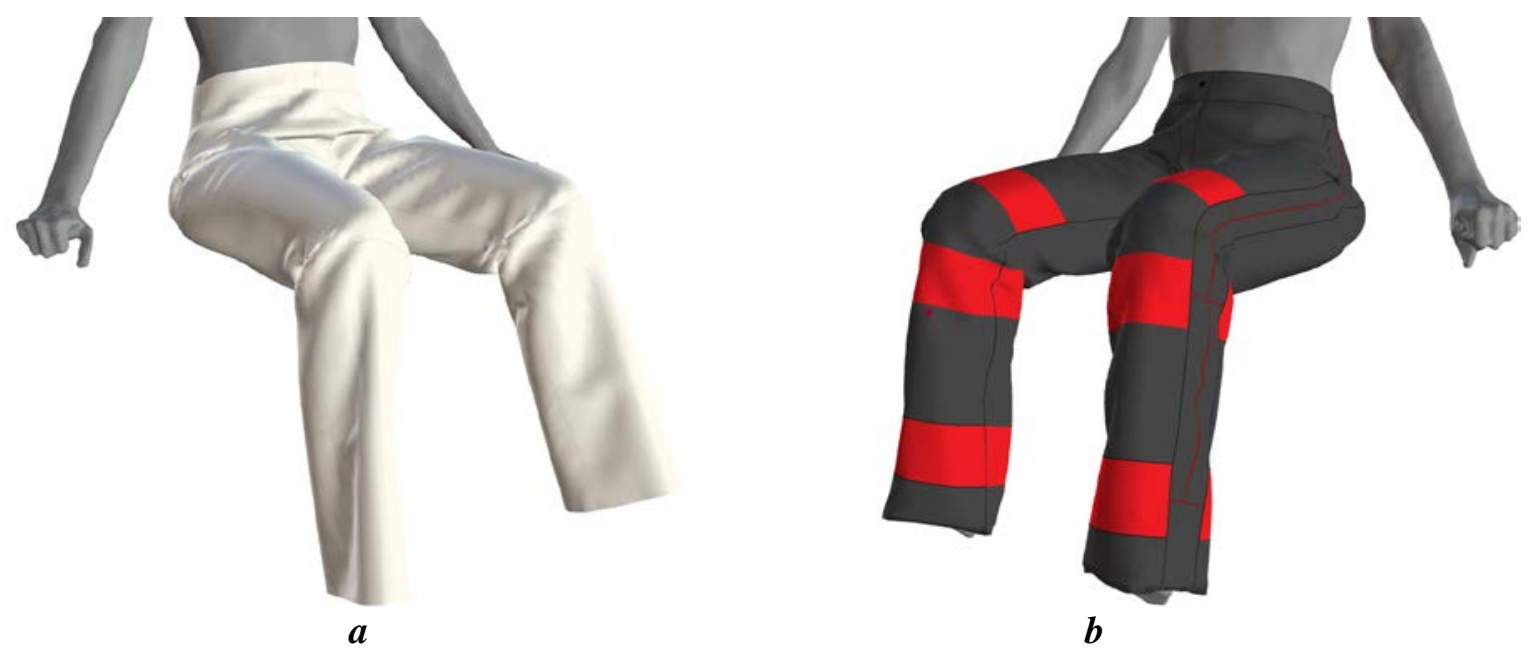

Figure 7. Virtual prototypes of trousers: a - regular; b - heated [20]

Because the quality of garment fit depends besides the accurate 3D body model also on the realistic fabric draping, within the theoretical part of the module the low-stress mechanical properties for fabric drape simulation in OptiTex PDS 3D software were 
presented. Measurement of fabrics' low-stress mechanical properties including tensile, shear, bending, surface and compression properties with FAST measuring system was presented, as well as their use for calculation of simulation of a physical model of a fabric and fabric behaviour during virtual prototyping.

The individual steps necessary for the creation of a garment virtual prototype using the 2D/3D Optitex PDS software are shown in the study material and supported in more detail with five videos (figure 8).

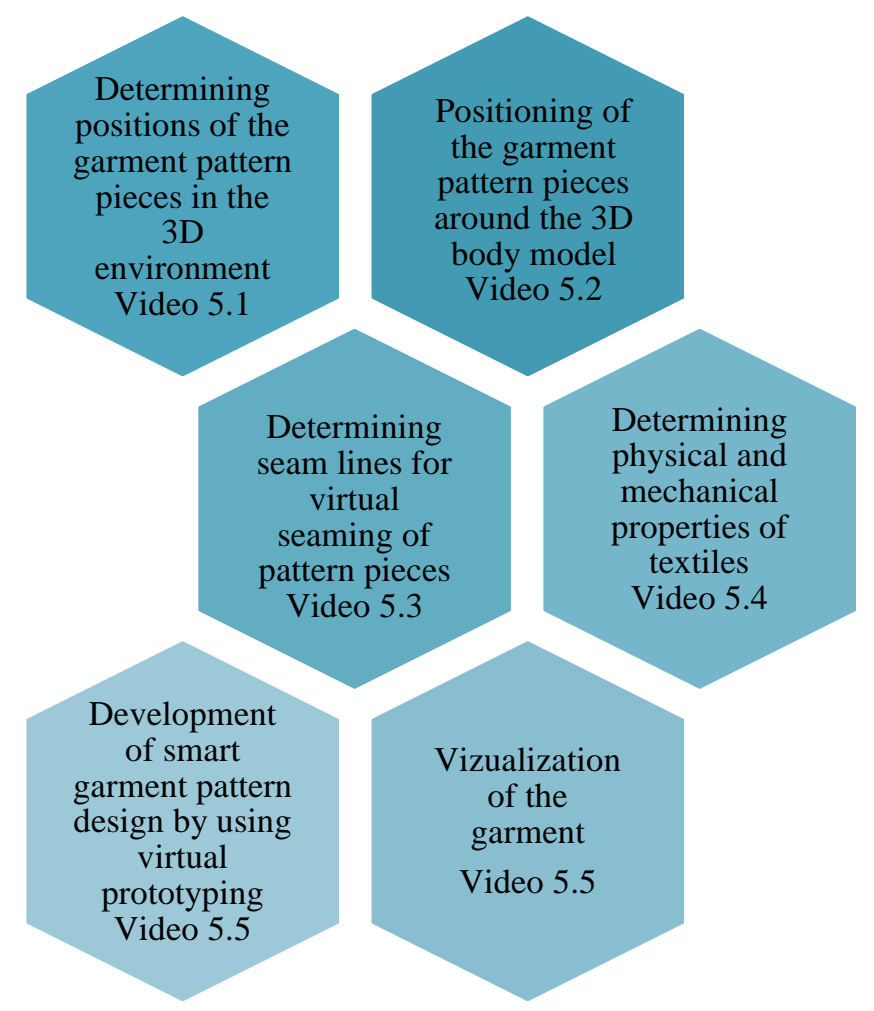

Figure 8. Steps for creation of a garment virtual prototype using 2D/3D Optitex PDS software

\section{CONCLUSIONS}

With the growing demand for customized products, e-commerce, and advances in virtual reality applications, virtual garment development is now in high demand to streamline the design and development processes of the apparel industry. Virtual garment prototyping has great potential in the fashion industry, especially by using adaptable digital body models to develop personalized garments for sports, medical and protective clothing. This contribution presents the development of kinematic 3D body models and their use for designing garments for different body postures. It also presents the topics of the module for the Erasmus+ project OptimTex, which is fully aligned with the new trends of digitalization.

\section{ACKNOWLEDGMENT}

The authors would like to acknowledge the support of the EC Programme Erasmus+, Project $n^{\circ}$ 2020-1-RO01-KA203-079823 “Software tools for textile creatives", acronym OptimTex. This project has been funded with support of the European Commission. This 
publication reflects the views only of the author, and the Commission cannot be held responsible for any use which may be made of the information contained therein.

\section{REFERENCES}

[1] Lay, R., Digital transformation - the ultimate challenge for the fashion industry, In: Perspectives, Available at: https://www2.deloitte.com/ [Accessed 10.7.2021]

[2] OptimTex project Application form, Erasmus+ KA2 - Strategic Partnership for Higher Education, Call 2020

[3] Stjepanović, Z., Rudolf, A., Jevšnik, S., Cupar, A., Pogačar, V., Geršak, J., 3D virtual prototyping of a ski jumpsuit based on a reconstructed body scan model, In: Buletinul Institutului Politehnic din Iaşi. Secția Textile, Pielarie, 2011, 57, 17-30

[4] Jevšnik, S., Pilar, T., Stjepanović, Z., Rudolf, A., Virtual prototyping of garments and their fit to the body, In: DAAAM International scientific book 2012, DAAAM International Publishing, Vienna, 2012, 601-618

[5] Rudolf, A., Cupar, A., Kozar, T., Stjepanović, Z., Study regarding the virtual prototyping of garments for paraplegics, In: Fibers Polym., 2015, 16, 1177-1192

[6] Bruniaux P, Cichocka A, Frydrych I., 3D Digital Methods of Clothing Creation for Disabled People, In: Fibres \& Textiles in Eastern Europe, 2016, 24, 5, 119, 125-131

[7] Naglić, M.M., Petrak, S., Stjepanović, Z., Analysis of 3D construction of tight fit clothing based on parametric and scanned body models, In: Proc. of 7th Int. Conf. on 3D Body Scanning Technologies, Lugano, Switzerland, 2016, 302-313

[8] Rudolf, A., Görlichová, L., Kirbiš, J., Repnik, J., Salobir, A., Selimović, I., Drstvenšek, I., New technologies in the development of ergonomic garments for wheelchair users in a virtual environment, In: Industria Texttila, 2017, 68, 2, 83-94, https://doi.org/10.35530/IT.068.02.1371

[9] Bogović, S., Stjepanović, Z., Cupar, A., Jevšnik, S., Rogina-Car, B., Rudolf, A., The Use of New Technologies for the Devel-opment of Protective Clothing: Comparative Analysis of Body Dimensions of Static and Dynamic Postures and its Application, In: Autex Research Journal, 2019, 19, 301-311

[10] Cupar, A., Stjepanović, Z., Olaru, S., Popescu, G., Salistean, A., Rudolf, A., CASP methodology applied in adapted garments for adults and teenagers with spine deformity, In: Industria Textila, 2019, 70, 5, 435-446, http://doi.org/10.35530/IT.070.05.1619

[11] Olaru, S., Popescu, G., Anastasiu, A., Mihăilă, G., Săliştean, A., Innovative concept for personalized pattern design of safety equipment, In: Industria Textila, 2020, 71, 1, 50-54, http://doi.org/10.35530/IT.071.01.1620

[12] Boulic, R., Magnenat-Thalmann, N., Thalmann, D., A global human walking model with realtime kinematic personification, In: The Visual Computer: International Journal of Computer Graphics. 1990, 6, 344-358

[13] Jung, M., Badler, N.I., Noma, T., Animated Human Agents with Motion Planning Capability for 3D-Space Postural Goals, In: Journal of Visualization and Computer Animation, 1994, 5, 225-246

[14] Kalra, P., Magnenat-Thalmann, N., Moccozet, L., Sannier, G., Aubel, A., Thalmann, D., Realtime animation of realistic virtual humans, In: IEEE Computer Graphics and Applications, 1998, 18, 42-56

[15] Leipner, A., Krzywinski, S., 3D Product Development Based on Kinematic Human Models, In: Proceedings of the 4th International Conference on 3D Body Scanning Technologies, Long Beach CA, USA, 19-20 November 2013; Editor: Hometrica Consulting, Switzerland; Publisher: Hometrica Consulting, Switzerland, 310-316

[16] Kozar, T., Rudolf, A., Cupar, A., Jevšnik, S., Stjepanovič, Z., Designing an adaptive 3D body model suitable for people with limited body abilities, In: Journal of Textile Science \& Engineering, 2014, 4, 5, 1-13

[17] Zhang, D., Krzywinski, S., Development of a Kinematic Human Model for Clothing and High Performance Garments, In Proc. of 3DBODY.TECH 2019 - 10th Int. Conf. and Exh. on 3D Body Scanning and Processing Technologies, Lugano, Switzerland, 22-23 Oct. 2019, 68-73 
[18] Zhang, D., Krzywinski, S., Development of a Kinematic Human Model for Clothing Design / simulation, AUTEX2019 - 19th World Textile Conference on Textiles at the Crossroads, 11-15 June 2019, Ghent, Belgium

[19] Klepser, A., Pirch, C., Is this real? Avatar Generation for 3D Garment Simulation, In: Journal of Textile \& Apparel Technology \& Management (JTATM), 2021, Special Issue, 12, 1-11

[20] Rudolf, A., Štampfer, J., Sadek, J., Surla-Pavlović, B., Hudournik, M., Kolanovič, V., Novak, J., Borovec, M., Belšak, R., Gotlih, K., Designing a smart heating trousers to improve wheelchair users legs thermal comfort (paper in review). 